Pacific Journal of Mathematics

GENERALIZED GOURSAT PROBLEM 


\section{GENERALIZED GOURSAT PROBLEM}

\section{Robert P. Holten}

1. Introduction. The linear first order system of rartial differential equations in two independent variables

$$
V_{x}^{i}=\sum_{\jmath=1}^{N} b_{i j}(x, y) V_{y}^{i}+\sum_{j=1}^{N} e_{i j}(x, y) V^{j}+f_{i}(x, y), \quad i=1, \cdots, N
$$

with coefficients that are continuous functions of the independent variables is hyperbolic at $(0,0)$ if there is a real matrix $T=\left(t_{i j}\right)$ non-singular with continuously differentiable components in some neighborhood of $(0,0)$ such that $T^{-1} B T$ is a diagonal matrix and $B=\left(b_{i j}\right)$. We consider problems of the following kind:

(1-2) To find such conditions that the hyperbolic system (1-1) has a unique solution which satisfies a number of linear equations along several arcs issuing from the origin.

Picard was probably the first to consider a non-analytic problem of this type [7]. Two types of hypotheses are needed for (1-2). The first is geometrical i.e. we require certain curves determined by the functions $b_{i j}$ (the characteristic curves) to intersect the arcs issuing from $(0,0)$ (the data arcs) in a manner described in $\S 2$ as Conditions (2.1). The second group of assumptions concern certain matrices made up from $b_{i j}, t_{i j}$ and the coefficients of the linear equations mentioned in (1-2) and the slopes of the data arcs at $(0,0)$. Some of these matrices are required to be non-singular and others to have eigenvalues with modulus $l_{\text {ass }}$ than one. In $\S 3$ we consider the case that all the data arcs lie between two consecutive characteristic curves through $(0,0)$. In this case we generalize the theorem proved in $\S 2$ by giving conditions for there to be a unique solution which is $C^{n}$. In $\S 5$ we state conditions under which the hypotheses of Theorem 3.1 can always be satisfied for sufficiently large $n$. We show at the end of $\S 5$ that if some of the hypotheses of Theorem 3.1 are omitted the solution (if it exists) is no longer unique. In $\S 6$ we solve a mixed problem for the general second order hyperbolic equation.

The equations (1-1) are simplified by the linear transformation

$$
U^{i}=\sum_{j=1}^{N} t_{i j} V^{j}
$$

Without loss of generality we consider the problem (1-2) in the reduced form.

Received October 31, 1960. 
2. Data arcs and characteristic curves. Under what conditions does a system of $N$ linear first order equations of the sort

(S)

$$
U_{x}^{i}+A^{i}(x, y) U_{y}^{i}=\sum_{j=1}^{N} E^{i j}(x, y) U^{j}+G^{i}(x, y) \quad i=1, \cdots, N
$$

and some linear combinations of $U^{1}, \cdots, U^{N}$ given along arcs issuing from a point

determine $U^{1}, \cdots, U^{N}$ uniquely?

We are concerned with real valued functions of real variables. Suppose the functions $A^{i}$ are $C^{1}$ (actually all the conditions we will impose need only hold in some neighborhood of $(0,0))$. Let $\gamma_{i}(x, y)$ be the curve passing through the point $(x, y)$ and which has slope $A^{i}(\xi, \eta)$ at every point $(\xi, \eta)$ on it. These are called the characteristic curves of (S). The equation for $\gamma_{i}(x, y)$ is $\eta=y^{i}(\xi ; x, y)$ where

$$
\begin{aligned}
& y_{\xi}^{i}(\xi ; x, y)=A^{i}\left(\xi, y^{i}(\xi ; x, y)\right) \\
& y^{i}(\xi ; \xi, \eta)=\eta .
\end{aligned}
$$

We come to the arcs along which we specify linear combinations of $U^{1}, \cdots, U^{N}$. Let $N_{0}$ be any positive integer less than $N+1$ and let $\mathrm{C}_{1}, \cdots, C_{N}, \widehat{C}_{1}, \cdots, C_{N_{0}-1}$ be curves issuing from $(0,0)$ which have continuously turning tangents. Let these curves be given non-parametrically by

$$
\begin{aligned}
& C_{i}: \quad y=\varphi_{i}(x) \quad \varphi_{i}(0)=0 \\
& i=1, \cdots, N \\
& \widehat{C}_{k}: \quad y=\widehat{\varphi}_{k}(x) \quad \widehat{\varphi}_{k}(0)=0 \\
& k=1, \cdots, N_{0}-1 \text {. }
\end{aligned}
$$

The conditions (2-1) below help determine whether the range for $x$ is either $x \geqq 0$ or $x \leqq 0$. Our problem (S) may be started more explicitly in terms of data ares:

(S)

$$
\left\{\begin{array}{l}
U_{x}^{i}+A^{i}(x, y) U_{y}^{i}=\sum_{j=1}^{N} E^{i j}(x, y) U^{j}+G^{i}(x, y) \\
\sum_{j=1}^{N} a_{i j}(x) U^{j}\left(x, \varphi_{i}(x)\right)=H_{i}(x), \quad i=1, \cdots, N \\
\sum_{j=1}^{N} \hat{a}_{k j}(x) U^{j}\left(x, \hat{\varphi}_{k}(x)\right)=\hat{H}_{k}(x), \quad k=1, \cdots, N_{0}-1 .
\end{array}\right.
$$

We seek solutions of (S) on closed domains, $R_{N_{0}}$, satisfying the following:

1. The boundary of $R_{N_{0}}$ is a piecewise smooth simply closed curve.

2. The origin is on the boundary of $R_{N_{0}}$ and $R_{N_{0}}$ contains a nonzero length segment of each data arc issuing from $(0,0)$.

(2-1) 3. For every $(x, y)$ in $R_{N_{0}}$ and $i<N_{0}, \gamma_{i}(x, y)$ intersects $C_{i}$ or $\hat{C}_{i}$ just once at a point we denote by $P_{i}(x, y)$. If $\gamma_{i}(x, y)$ intersects both 
$C_{i}$ and $\hat{C}_{i}$, then the point of intersection is $(0,0)$. For $i \geqq N_{0}$ and every $(x, y)$ in $R_{N_{0}}, \gamma_{i}(x, y)$ intersects $C_{i}$ just once at $P_{i}(x, y)$.

4. For each $(x, y)$ in $R_{N_{0}}$, the entire segment of $\gamma_{i}(x, y)$ from $(x, y)$ to $P_{i}(x, y)$ lies in $R_{N_{0}}$.

We assume temporarily that there are domains $R_{N_{0}}$ which satisfy these conditions and which have small as we please diameter. Later in this paper we discuss the existence of these domains. Loosely speaking the subscript $N_{0}$ has the significance that $N_{0}-1$ characteristic curves issue from $(0,0)$ into the interior of $R_{N_{0}}$ and as we will see consequently linearly combinations must be given along $N+N_{0}-1$ arcs.

Notice that if $N_{0}>\mathbf{1}$, (S) over determines the values of a solution at $(0,0)$. We suppose $(S)$ is consistent at $(0,0)$. That is, there are numbers $b_{i}, c_{i}, d_{i}$ (to be interpreted as $U^{i}(0,0), U_{x}^{i}(0,0), U_{y}^{i}(0,0)$ ) which satisfy the equations:

$$
\begin{aligned}
& \sum_{j=1}^{N} a_{i j}(0) b_{j}=H_{i}(0) \\
& \sum_{j=1}^{N} \widehat{a}_{k \jmath}(0) b_{j}=\hat{H}_{k}(0)
\end{aligned}
$$

and

$$
\begin{aligned}
\sum_{j=1}^{N} a_{i j}(0)\left[\mathscr{P}_{i}^{1}(0)-A^{j}(0,0)\right] d_{j}= & -\sum_{j=1}^{N} a_{i j}(0)\left[\sum_{m=1}^{N} E^{j m}(0,0) b_{m}+G^{j}(0,0)\right] \\
& -\sum_{j=1}^{N} a_{i j}^{1}(0) b_{j}+H_{i}^{1}(0)
\end{aligned}
$$

and same equation with $i, a, \varphi, H$ replaced respectively by $k, \hat{a}, \hat{\varphi}, \hat{H}$

and

$$
c_{i}+A^{i}(0,0) d_{i}=\sum_{j=1}^{N} E^{i j}(0,0) b_{j}+G^{i}(0,0) .
$$

Certain matrices play an important role in what follows. Let $Q(n)$ be the square $N \times N$ matrix such that

$$
\begin{aligned}
& Q(n)_{i i}=0 \\
& Q(n)_{i j}=\max \left\{\left|\frac{a_{i j}(0)}{a_{i i}(0)}\right| \cdot\left|\frac{\varphi_{i}^{1}(0)-A^{j}(0,0)}{\varphi_{i}^{1}(0)-A^{i}(0,0)}\right|^{n}\left|\frac{\widehat{a}_{i j}(0)}{\hat{a}_{i i}(0)}\right| \cdot\left|\frac{\hat{\varphi}_{2}^{1}(0)-A^{j}(0,0)}{\hat{\varphi}_{2}^{\prime}(0)-A^{i}(0,0)}\right|^{n}\right\} \\
& i=1, \cdots, N_{0}-1 \text { and } j=1, \cdots, N \\
& Q(n)_{i j}=\left|\frac{a_{i j}(0)}{a_{i i}(0)}\right| \cdot\left|\frac{\varphi_{i}^{1}(0)-A^{j}(0,0)}{\varphi_{i}^{1}(0)-A^{i}(0,0)}\right|^{n} \\
& i=N_{0}, \cdots, N \text { and } j=1, \cdots, N .
\end{aligned}
$$


We assume that the slopes of $C_{i}$ and $\hat{C}_{i}$ differ from the slope of $\gamma_{i}(0,0)$ at $(0,0)$. That is, $\varphi_{i}^{1}(0) \neq A^{i}(0,0)$ and $\varphi_{k}^{1}(0) \neq A^{k}(0,0)$. We also assume that $a_{i i}(0) \neq 0$.

Let $M(n)$ be the $N \times N$ matrix such that

$$
M(n)_{i j}=a_{i j}(0) \cdot\left(\varphi_{i}^{1}(0)-A^{j}(0,0)\right)^{n} .
$$

Let $\hat{M}(n)$ be the $N \times\left(N_{0}-1\right)$ matrix such that

$$
\widehat{M}(n)_{i j}=\hat{a}_{i j}(0)\left(\hat{\phi}_{i}^{1}(0)-A^{j}(0,0)\right)^{n}, \quad i=1, \cdots, N_{0}-1 .
$$

Let $\bar{M}(n)$ be the compound $N \times\left(N_{0}-1\right)$ matrix

$$
\bar{M}(n)=\left(\begin{array}{l}
M(n) \\
\hat{M}(n)
\end{array}\right) .
$$

For any matrix $P$ let $\bar{\lambda}(P)$ be the maximum modulus of all the eigenvalues of $P$.

LEMMA 2.1. If $\bar{\lambda}(Q(n))<1$, then $M(n)$ is nonsingular.

Proof. Suppose $M(n)$ is singular. There is then a nonzero vector $x$ such that $M(n) x=0$. That is

$$
\begin{gathered}
\sum_{j=1}^{N} a_{i j}(0)\left[\varphi_{i}^{1}(0)-A^{\jmath}(0,0)\right]^{n} x_{j}=0 \\
\left|a_{\imath i}(0)\right| \cdot\left|\varphi_{i}^{\mathrm{1}}(0) A^{i}(0,0)\right|^{n}\left|x_{i}\right| \leqq \sum_{\substack{j=1 \\
j \neq i}}^{N}\left|a_{i j}(0) \| \varphi_{i}^{1}(0)-A^{\mathcal{J}}(0,0)\right|^{n}\left|x_{\jmath}\right| .
\end{gathered}
$$

Dividing by $\left|a_{i i}(0)\right| \cdot\left|\varphi_{i}^{1}(0)-A^{i}(0,0)\right|^{n}$ we get

$$
\left|x_{i}\right| \leqq \sum_{j=1}^{N} Q(n)_{i j}\left|x_{j}\right|
$$

Let $|x|$ be the vector whose $i$ th component is $\left|x_{i}\right|$ then $|x| \leqq Q(n)|x|$ the inequality is understood to hold for each pair of corresponding components. Hence

$$
(p+1)|x| \leqq \sum_{k=0}^{p} Q^{k}(n)|x|
$$

so that $\sum_{k=0}^{p} Q^{k}(n)|x|$ diverges as $p \rightarrow \infty$. Therefore $\bar{\lambda}(Q(n)) \geqq 1$.

THEOREM 2.1. If $(\mathbf{S})$ is consistent at $(0,0)$ and all its given functions are $C^{1}$ and $\bar{\lambda}(Q(0))<1$ and $\bar{\lambda}(Q(1))<1$, then on some $R_{N_{0}}$ there is a unqiue $C^{1}$ solution of $(\mathrm{S})$.

Proof. We construct the solution by iteration. Let ${ }^{0} U^{i}(x, y)=$ $b_{i}+c_{i} x+d_{i} y$ and obtain ${ }^{s+1} U^{1}, \ldots,{ }^{s+1} U^{N}$ from ${ }^{s} U^{1}, \ldots,{ }^{s} U^{N}$ using 


$$
{ }^{s+1} U_{x}^{i}+A^{i}(x, y)^{s+1} U_{y}^{i}=\sum_{j=1}^{N} E^{i j}(x, y)^{s} U^{j}+G^{i}(x, y)
$$

and

$$
{ }^{s+1} U^{i}\left(x, \varphi_{i}(x)\right)=-\sum_{\substack{j \neq 1 \\ j \neq i}}^{N} \frac{a_{i j}(x)}{a_{i i}(x)}{ }^{s} U^{j}\left(x_{1} \varphi_{i}(x)\right)+\frac{1}{a_{i i}(x)} H_{i}(x)
$$

and

$$
{ }^{s: 1} U^{k}\left(x, \widehat{\varphi}_{k}(x)\right)=-\sum_{\substack{j=1 \\ j \neq k}}^{N} \frac{\widehat{a}_{k j}(x)}{\widehat{a}_{k k}(x)} U^{s}\left(x, \widehat{\varphi}_{k}(x)\right)+\frac{1}{\hat{a}_{k k}(x)} \hat{H}_{k}(x) .
$$

Equivalently,

$$
\begin{array}{r}
{ }^{s+1} U^{i}(x, y)={ }^{s+1} U^{i}\left(P_{i}(x, y)\right)+\int_{P_{i}(x, y)}^{(x, y)}\left[\sum_{j=1}^{N} E^{\imath \jmath}(\xi, \eta)^{s} U^{j}(\xi, \gamma)\right. \\
\left.G^{i}(\xi, \eta)\right] d \xi .
\end{array}
$$

IWhere the integral is taken along $\gamma_{i}(x, y)$ and $P_{i}(x, y)$ is the intersection of $\gamma_{\imath}(x, y)$ with $C_{i} \cup \hat{C}_{i} \mid$ and for $P_{\imath}(x, y)$ on $C_{\imath}$

$$
{ }^{s+1} U^{i}\left(P_{i}(x, y)\right)=-\sum_{\substack{j=1 \\ j \neq i}}^{N} \frac{a_{i j}\left(\alpha^{2}(x, y)\right)}{a_{\imath \imath}\left(\alpha^{i}(x, y)\right)} U^{s}\left(P_{i}(x, y)\right)+\frac{1}{a_{i i}\left(\alpha^{i}(x, y)\right)} H_{i}\left(\alpha^{i}\right)
$$

and for $P_{\imath}(x, y)$ an $C_{i}$

$$
{ }^{s+1} U^{i}\left(P_{i}(x, y)\right)=-\sum_{\substack{j=1 \\ j \neq i}}^{N} \frac{\hat{a}_{i j}\left(\alpha^{i}\right)}{\hat{a}_{i i}\left(\alpha^{i}\right)} U^{j}\left(P_{i}\right)+\frac{1}{\hat{a}_{i i}\left(\alpha^{j}\right)} \hat{H}_{i}\left(\alpha^{i}\right)
$$

where $\alpha^{i}(x, y)$ is the abscissa of $P_{i}(x, y)$.

From the assumption that $(\mathrm{S})$ is consistent at $(0,0)$ we can conclude that ${ }^{s+1} U^{i}\left(P_{i}(x, y)\right)$ is properly defined when $P_{i}(x, y)$ is the origin [i.e. when $(x, y)$ lies on $\left.\gamma_{i}(0,0)\right]$. It is easy to see that

$$
\left\|{ }^{s+2} U^{i}-{ }^{s+1} U^{i}\right\| \leqq \sum_{j=1}^{N} T_{i j}\left\|{ }^{s+1} U^{j}-{ }^{s} U^{j}\right\|
$$

where $\left|T_{i j}-Q(0)_{i j}\right|$ can be made as small as we please by taking the diameter of $R_{N_{0}}$ small enough. Since $\bar{\lambda}(Q(0))<1$ we conclude that ${ }^{0} U^{i}$, ${ }^{1} U^{i}, \cdots,{ }^{s} U^{i}, \cdots$ converges uniformly for each $i=1, \cdots, N$. That there is at most one solution follows also immediately. The proof that the first partial derivatives also converge uniformly depends in the following way on the fact that $\bar{\lambda}(Q(1))<1$ :

By taking the $y$-partial derivative of (2-3) and (2-4) and using (2-2) to eliminate ${ }^{s+1} U_{x}^{i}$ we have

$$
\left[\varphi_{i}^{1}(x)-A^{i}\left(x, \varphi_{i}(x)\right)\right]^{s+1} U_{y}^{i}\left(x, \varphi_{i}(x)\right)
$$




$$
\begin{aligned}
= & \sum_{\substack{j=1 \\
j \neq i}}^{N} \frac{a_{i j}(x)}{a_{i i}(x)}\left[\varphi_{i}^{1}(x)-A^{j}\left(x, \varphi_{i}(x)\right)\right]^{s} U_{y}^{j}\left(x, \varphi_{i}(x)\right) \\
& + \text { terms not involving derivatives of } U
\end{aligned}
$$

and

$$
\begin{aligned}
{ }^{s+1} U_{y}^{i}(x, y)= & {\left[\varphi_{i}^{1}\left(\alpha^{i}\right)-A^{i}(x, y)\right] \cdot \alpha_{y}^{i}(x, y) \cdot{ }^{s+1} U_{y}^{i}\left(\alpha^{i}, \varphi_{i}\left(\alpha^{i}\right)\right) } \\
& +\int_{\alpha^{i}}^{x} \sum_{j=1}^{N} E^{i j}(\xi, \eta) \cdot{ }^{s} U_{y}^{j}(\xi, \eta) \cdot y_{y}^{i}(\xi ; x, y) \cdot d \xi \\
& + \text { lower order terms. }
\end{aligned}
$$

It is not hard to show that

$$
\alpha_{y}^{i}(0,0)=\frac{1}{\varphi_{i}^{1}(0)-A^{i}(0,0)}
$$

therefore

$$
\left[\varphi_{i}^{1}\left(\alpha^{i}\right)-A^{i}(x, y)\right] \alpha_{y}^{i}
$$

has the limit 1 as $(x, y)$ approaches $(0,0)$. Consequently considering both (2-4) and (2-5) we have

$$
\begin{aligned}
\left\|{ }^{s+1} U_{y}^{i}-{ }^{s} U_{y}^{i}\right\| \leqq & \sum_{j=1}^{N}\left(Q_{i j}(1)+\varepsilon_{1}\right)\left(1+\varepsilon_{2}\right)\left\|{ }^{s} U_{y}^{j}-{ }^{s-1} U_{y}^{j}\right\| \\
& +\beta \sum_{j=1}^{N}\left\|s U^{\jmath}-{ }^{s-1} U^{j}\right\|
\end{aligned}
$$

where $\varepsilon_{1}$ and $\varepsilon_{2}$ approach zero as the diameter of $R_{N_{0}}$ approaches zero. $\beta$ is some fixed constant. Since $\bar{\lambda}(Q(1))<1$ by selecting $R_{N_{0}}$ with small enough diameter the eigenvalues of the matrix $L$ where

$$
L_{i j}=\left(Q_{i j}(1)+\varepsilon_{1}\right)\left(1+\varepsilon_{2}\right)
$$

also have modulus less than one. Let

$$
v_{s}^{i}=\left\|{ }^{s} U_{y}^{i}-{ }^{s-1} U_{y}^{i}\right\| \text { and } u^{i}=\left\|{ }^{1} U^{i}-{ }^{0} U^{i}\right\|
$$

them

$$
v_{s+1} \leqq L v_{s}+\beta T^{s} u
$$

where the inequality must hold between pairs of corresponding components. It is easily seen that

$$
\sum_{s=1}^{\infty} v_{s} \leqq(1-L)^{-1} v_{1}+\beta(1-L)^{-1}(1-T)^{-1} u
$$

and our convergence is assured.

In [4] Meltzer assumed that there are only two data arcs. The 
method used in [5] by Mihailow permits the characteristic curves and data arcs only to be straight lines. In [3] the author obtained results in the large by making more assumptions relating the slopes of the data arcs and the characteristic curves. In [6] Peyser in effect requires that $N-1$ of the data arcs be identical and consequently the matrices $Q(0)$ and $Q(1)$ are nilpotent. Finally in [9] Yosida assumes that the matrix $M(0)$ is diagonal and consequently $Q(0)$ is the zero matrix.

3. Higher order solutions. In this section we prove a generalization of Theorem 2.1 for $N_{0}=1$. This is the case that all the data arcs lie between two consecutive characteristic curves through the origin. With the addition of a consistency hypothesis for higher order derivatives at $(0,0)$ the generalization when $N_{0}>1$ is also true. We begin by proving a lemma about

$$
\left\{\begin{array}{l}
U_{x}^{i}+A^{i}(x, y) U_{y}^{i}=F^{i}(x, y) \\
\sum_{j=1}^{N} a_{i j}(x) U^{j}\left(x, \varphi_{i}(x)\right)=H_{i}(x), \quad i=1, \cdots, N
\end{array}\right.
$$

[this is (S) with $N_{0}=1$ and $E^{i j}=0$ ]

Lemma 3.1. If $n$ is any nonnegative integer and $A^{i}, F^{i}, H^{i}, a_{i j}, \varphi_{i}$ are $C^{n+1}$ and $\bar{\lambda}(Q(n))<1$ and $\bar{\lambda}(Q(n+1))<1$ and $M(0), \cdots, M(n-1)$ each have rank $N$ (i.e., are nonsingular), then on some $R_{1}$ (we assume that such domains exist) there is exactly one $C^{n+1}$ solution of $\left(\mathbf{S}_{0}\right)$. Moreover $R_{1}$ depends on neither $F^{i}$ nor $H_{i}$.

Proof. As we did before we perform the iteration:

$$
\begin{aligned}
{ }^{s+1} U_{x}^{i}+A^{i}(x, y)^{s+1} U_{y}^{i} & =F^{i}(x, y) \\
{ }^{s+1} U^{i}\left(x, \varphi_{i}(x)\right) & =-\sum_{\substack{j=1 \\
j \neq i}}^{N} \frac{a_{i j}(x)}{a_{i i}(x)}{ }^{s} U^{j}\left(x, \varphi_{i}(x)\right)+\frac{1}{a_{i i}(x)} H_{i}(x) .
\end{aligned}
$$

Taking the $n$th derivative of the second equation we get

$$
\begin{aligned}
& \sum_{p=0}^{n} s+1 U_{p, n-p}^{i}\left(x, \varphi_{i}(x)\right)\left(\begin{array}{c}
n \\
n-p
\end{array}\right)\left[\varphi_{i}^{1}(x)\right]^{n-p} \\
& =-\sum_{\substack{j=1 \\
j=i}}^{N} \frac{a_{i j}}{a_{i i}} \sum_{p=0}^{n} s U_{p, n-p}^{j}\left(x, \varphi_{i}(x)\right)\left(\begin{array}{c}
n \\
n-p
\end{array}\right)\left[\varphi_{i}^{1}(x)\right]^{n-p}
\end{aligned}
$$

+ terms involving derivatives of order less than $n$ of ${ }^{s+1} U$ and ${ }^{s} U$, where

$$
U_{p, n-p}^{i}=\frac{\partial^{n} U^{i}}{\partial x^{p} \partial y^{n-p}} \text { and }\left(\begin{array}{c}
n \\
n-p
\end{array}\right)=\frac{n !}{p !(n-p) !} .
$$

Using the first equation we have 


$$
{ }^{8} U_{p, n-p}^{i}=\left[-A^{i}(x, y)\right]^{p s} U_{o, n}^{i}
$$

+ terms involving derivatives of ${ }^{s} U$ of order less than $n$.

Consequently,

$$
\begin{aligned}
\left\lceil\varphi_{i}^{1}(x)\right. & \left.-A^{i}\left(x, \varphi_{i}(x)\right)\right]^{n s+1} U_{0, n}^{i}\left(x, \varphi_{i}(x)\right) \\
& =\sum_{\substack{j=1 \\
j \neq i}}^{N} \frac{a_{i j}(x)}{a_{i i}(x)}\left[\varphi_{i}^{1}(x)-A^{j}\left(x, \varphi_{i}(x)\right)\right]^{n} s U_{0, n}^{j}\left(x, \varphi_{i}(x)\right)
\end{aligned}
$$

+ terms of order less than $n$.

Since $M(0), \cdots, M(n-1)$ and $M(n), M(n+1)$ are nonsingular (see Lemma 2.1) the values of any solution of $\left(\mathrm{S}_{0}\right)$ and all their derivatives up to and including order $(n+1)$ are uniquely determined at $(0,0)$. Let $c_{i}\left(p_{1}, p_{2}\right)$ be the value determined for $U_{p_{1}, p_{2}}^{i}(0,0)$. We begin our iteration with

$$
{ }^{0} U^{i}(x, y)=\sum_{0 \leqq p_{1}+p_{2} \leqq n+1} c_{2}\left(p_{1}, p_{2}\right) \cdot \frac{x^{p_{1}} \cdot y^{p_{2}}}{p_{1} ! p_{2} !}
$$

It follows that

$$
{ }^{s} U_{p_{1}, p_{2}}^{i}(0,0)=c_{i}\left(p_{1}, p_{2}\right) \text { for } 0 \leqq p_{1}+p_{2} \leqq n+1, s>0 .
$$

Now since $\bar{\lambda}(Q(n))<1$ we see that all the $n$th order derivatives of the sequence ${ }^{0} U^{i}, \cdots,{ }^{s} U^{i}, \cdots$ converge uniformly on some $R_{1}$ of sufficiently small diameter. Also it is clear that the functions $F^{i}$ and $G^{i}$ are not involved in how small the diameter must be chosen. That there is at most one solution which is $C^{n}$ follows in the customary way. It remains to see that we have a $C^{n+1}$ solution to $\left(\mathrm{S}_{0}\right)$.

Since

$$
{ }^{s} U_{p, n+1-p}^{i}=\left[-A^{i}(x, y)\right]^{p s} U_{0, n+1}^{i}
$$

+ terms involving derivatives of ${ }^{s} U$ of order less than $n+1$, we need consider only the convergence of

$$
{ }^{0} U_{0, n+1}^{i}, \cdots,{ }^{s} U_{0, n+1}^{i}, \cdots
$$

Now

$$
\begin{aligned}
{ }^{s+1} U^{i}(x, y) & ={ }^{s+1} U^{i}\left(P_{i}(x, y)\right)+\int_{P_{i}(x, y)}^{(x, y)} F^{i}(\xi, \eta) d \xi \\
{\left[P_{i}(x, y)=\right.} & \text { the point } \left.\left(\alpha^{i}(x, y), \varphi_{i}\left(\alpha^{i}(x, y)\right)\right)\right] \\
{ }^{s+1} U_{0 . n+1}^{i}= & {\left[\alpha_{y}^{i}\right]^{n+1} \sum_{p=0}^{n+1}\left[\varphi_{i}^{1}\right]^{n+1-p} s^{s+1} U_{p, n+1-p}^{i}\left(\begin{array}{c}
n+1 \\
p
\end{array}\right) } \\
& + \text { terms of order less than } n+1 .
\end{aligned}
$$




$$
\begin{gathered}
\alpha_{y}^{i}(0,0)=\frac{1}{\varphi_{i}^{1}(0)-A^{i}(0,0)}, \\
{\left[\alpha _ { y } ^ { i } \left[{ }^{n+1}\left[\varphi_{i}^{1}-A^{i}\right]^{n+1}\right.\right.}
\end{gathered}
$$

can be made as near 1 as we please by taking the diameter of $R_{1}$ small enough. From this observation and the assumption $\bar{\lambda}(Q(n+1))<1$ the series

$$
{ }^{0} U_{0, n+1}^{i}, \cdots,{ }^{s} U_{0, n+1}^{i}, \cdots
$$

converge uniformly. Consequently we have indeed the unique $C^{n+1}$ solution of $\left(\mathbf{S}_{0}\right)$.

For the system

$$
\begin{cases}U_{x}^{i}+A^{i}(x, y) U_{y}^{i}=\sum_{j=1}^{N} E^{i j}(x, y) U^{j}+G^{i}(x, y) & \\ \sum_{j=1}^{N} a_{i j}(x) U^{j}\left(x, \mathscr{P}_{i}(x)\right)=H_{i}(x), & i=1, \cdots, N\end{cases}
$$

we have

THEOREM 3.1. If $n$ is a nonnegative integer and $A^{i}, E^{i j}, G^{i}, a_{\imath, j}, \varphi_{i}$, $H_{\imath}$ are $C^{n+1}$ and $\bar{\lambda}(Q(n))<1$ and $\bar{\lambda}(Q(n+1))<1$ and $M(0), \cdots, M(n-1)$ each are nonsingular, then on some $R_{1}$ there is exactly one $C^{n+1}$ solution of $\left(S_{1}\right)$.

Proof. Using Lemma 3.1 we can define a sequence of functions which are $C^{n+1}$ on some $R_{1}$ as follows:

$$
\begin{gathered}
{ }^{s+1} U_{x}^{i}+A^{i}(x, y)^{s+1} U_{y}^{i}=\sum_{j=1}^{N} E^{i j}(x, y)^{s} U^{j}+G^{i}(x, y) \\
\sum_{j=1}^{N} a_{i j}(x)^{s+1} U^{j}\left(x, \varphi_{i}(x)\right)=H_{i}(x) .
\end{gathered}
$$

We can show that all the $(n+1)$ th order derivatives converge on some possibly smaller $R_{1}$. Using the same kind of calculations as before it is easy to see that

$$
\begin{aligned}
\left\|{ }^{s+2} U_{0, n+1}^{i}-{ }^{s+1} U_{0, n+1}^{i}\right\| \leqq & \sum_{j=1}^{N} T_{i \jmath}\left\|{ }^{s+2} U_{0, n+1}^{j}-{ }^{s+1} U_{0, n+1}^{j}\right\| \\
& +\sum_{j=1}^{N} S_{i j}\left\|{ }^{s+1} U_{0, n+1}^{j}-{ }^{s} U_{0, n+1}^{j}\right\|
\end{aligned}
$$

where $\bar{\lambda}(T)<1, T_{i j} \geqq 0$ and by taking the diameter of $R_{1}$ small enough each $S_{i j}$ can be made arbitrarily close to zero. We have in vectors

$V_{s+1} \leqq T \cdot V_{s \nmid 1}+S \cdot V_{s}$ (the inequality must hold for each component).

$$
(I-T) V_{s+1} \leqq S \cdot V_{s} \text {. }
$$


Since $(I-T)^{-1}=I+T+T^{2}+\cdots,(I-T)_{i j}^{-1} \geqq 0, V_{s+1} \leqq(I-T)^{-1} S V_{s}$. By choosing $R_{1}$ small enough

$$
\bar{\lambda}\left((I-T)^{-1} S\right)<1 .
$$

Except for this the proof of Theorem 3.1 is like Lemma 3.1.

4. Constructing the domains of dependence. We discuss this topic only for the case that the data arcs and characteristic curves are straight lines. The subject has been treated more completely in [3].

Suppose $\varphi_{i}(x)=m_{i} x, \hat{\varphi}_{k}(x)=\hat{m}_{k} x$ and $A^{i}$ are constant. By possibly renaming the variables we can assume

$$
A^{1} \leqq A^{2} \leqq \cdots \leqq A^{N}
$$

Let $\bar{R}_{N_{0}}$ be the region lying below both the line $\gamma_{N}(0,0)$ and the line $\gamma_{N_{0}}(0,0)$. $\quad R_{N_{0}}$ will be a part of $\bar{R}_{N_{n}}$.

Assume that all the data arcs lie in $\bar{R}_{N_{0}}$. Let the first $l$ data arcs lie to the left of the $y$-axis (i.e. $\varphi_{1}, \cdots, \varphi_{l}$ defined only for $x \leqq 0$, of course $l$ may be zero) and suppose

$$
A^{N} \geqq m_{1} \geqq \cdots \geqq m_{l} \text {. }
$$

We suppose the remaining data arcs are ordered so that

$$
m_{l+1} \leqq m_{l+2} \leqq \cdots \leqq m_{N} \leqq \hat{m}_{1} \leqq \cdots \leqq \hat{m}_{N_{0}-1} \leqq A^{N_{0}} \text {. }
$$

We further assume that

$$
\begin{aligned}
& m_{i}>A^{i}, i=1, \cdots, l \\
& m_{\imath}<A^{i}, i=l+1, \cdots, N \\
& \hat{m}_{k}>A^{k}, k=1, \cdots, N_{0}-1 .
\end{aligned}
$$

These last assumptions assure us that $C_{i}$ lies below $\gamma_{i}(0,0)$ and $\hat{C}_{k}$ lies above $\gamma_{k}(0,0)$.

Our final assumption excludes the possibility that by omitting some data arcs a domain $R_{M_{0}}$ satisfying conditions (2.1) can be constructed with $M_{0}<N_{0}$. This final assumption is: For $N_{0} \neq 1$ assume

$$
m_{N}>A^{1} \text { and } \hat{m}_{k}>A^{k+1}, k=1, \cdots, N_{0}-2 \text {. }
$$

We construct the domains $R_{N_{0}}$ for $N_{0} \neq 1$. The case $N_{0}=1$ offers no new difficulties. We begin by choosing a point $P_{0}$ with negative abscissa on $\gamma_{N}(0,0)$ (we exclude the special case that $C_{N-1}$ lies along $\left.\gamma_{1}(0,0)\right)$. Define points $p_{1}, \cdots, p_{N}$ as follows:

$$
p_{i}=\text { the intersection of } \gamma_{i}\left(p_{i-1}\right) \text { with } C_{i}, i=1, \cdots, N \text {. }
$$

Since $C_{n}$ lies above $\gamma_{1}(0,0)$ and $\hat{C}_{k}$ above $\gamma_{k+1}(0,0)$, we can continue 
with points $\hat{p}_{1}, \cdots, \hat{p}_{N_{0}}$ :

$$
\begin{aligned}
& \hat{p}_{1}=\text { the intersection of } \gamma_{1}\left(p_{N}\right) \text { with } \hat{C}_{1} \\
& \hat{p}_{k}=\text { the intersection of } \gamma_{k}\left(\hat{p}_{k-1}\right) \text { with } \hat{c}_{k}, k,=2, \cdots, N_{0}-1 \\
& \hat{p}_{N_{0}}=\text { the intersection of } \gamma_{\bar{N}}\left(\hat{p}_{N_{0}}-1\right) \text { with } \gamma_{N_{0}}(0,0)
\end{aligned}
$$

where $A<A^{\bar{N}}<A^{N_{0}}$ and no $A^{i}$ exists such that $A^{\bar{N}}<A^{i}<A^{N_{0}}$.

The boundary of $R_{N_{0}}$ consists of $\gamma_{N_{0}}(0,0)$ from $(0,0)$ to $p_{0}, \gamma_{1}\left(p_{0}\right)$ from $p_{0}$ to $p_{1}, \cdots, \gamma_{N}\left(p_{N-1}\right)$ from $p_{N-1}$ to $p_{N}, \gamma_{1}\left(p_{N}\right)$ from $p_{N}$ to $p_{1}, \gamma_{2}\left(p_{1}\right)$ from $\hat{p}_{1}$ to $\hat{p}_{2}, \cdots, \gamma_{N_{0}-1}\left(\hat{p}_{N_{0}-2}\right)$ from $\hat{p}_{N_{0}-2}$ to $\hat{p}_{N_{0}-1}, \gamma_{\bar{N}}\left(\hat{p}_{N_{0}-1}\right)$ from $\hat{p}_{N_{0}-1}$ to $\hat{p}_{N_{0}}, \gamma_{N_{0}}(0,0)$ from $\hat{p}_{N_{0}}$ to $(0,0)$. The domains $R_{N_{0}}$ constructed in this way satisfy conditions (2.1). Also, if we let $p_{0}$ approach $(0,0)$ along $\gamma_{N}(0,0)$ the diameter of $R_{N_{0}}$ approaches zero.

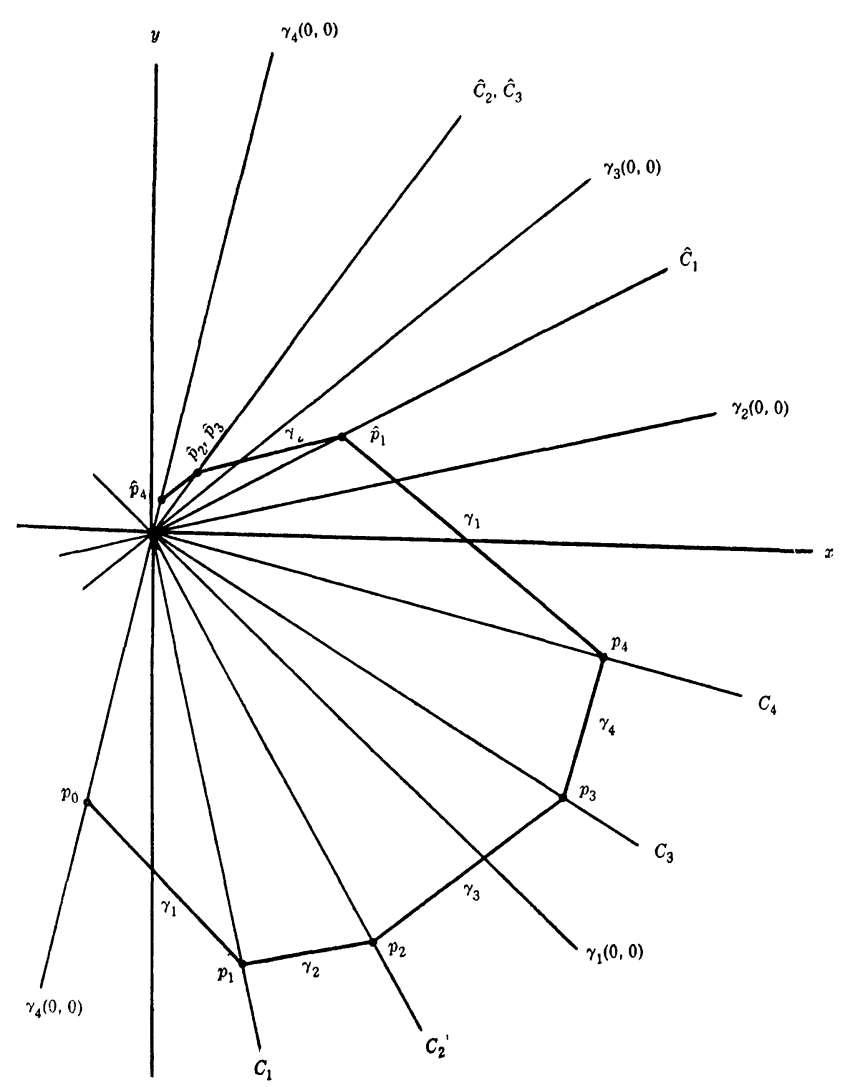

Fig.

5. Certain special systems. We turn our attention to the hypotheses of Theorem 3.1. We have $N_{0}=1$, that is, all the data arcs lie between two consecutive characteristic curves through $(0,0)$. In this case we call a system $\left(\mathrm{S}_{1}\right)$ regular if 


$$
A^{1}(0,0) \neq A^{j}(0,0) \text { and } \varphi_{i}^{1}(0) \neq \varphi_{j}^{1}(0) \text { for } i \neq j .
$$

For a regular system we can suppose without loss of generality that

$$
\begin{aligned}
A^{1}(0,0) & \leqq \varphi_{2}^{1}(0)<\varphi_{3}^{1}(0)<\cdots<\varphi_{N}^{1}(0)<\varphi_{1}^{1}(0) \\
& \leqq A^{2}(0,0)<A^{3}(0,0)<\cdots<A^{N}(0,0) .
\end{aligned}
$$

We can show, in case $\left(\mathrm{S}_{1}\right)$ is regular, that there is always an $\bar{n}$ such that $\bar{\lambda}(Q(n))<1$ whenever $n>\bar{n}$.

Recall that for $i \neq j$

$$
\begin{aligned}
& Q(n)_{i j}=\left|\frac{a_{i j}(0)}{a_{i i}(0)}\right|\left|\frac{\varphi_{i}^{1}(0)-A^{\jmath}(0,0)}{\varphi_{i}^{1}(0)-A^{i}(0,0)}\right|^{n} \\
& Q(n)_{i i}=0 .
\end{aligned}
$$

The eigenvalues of $Q(n)$ satisfy the equation $\operatorname{Det}(Q(n)-\lambda I)=0$.

LEMmA 5.1. If $d_{1}<d_{2}<\cdots<d_{N} \leqq e_{1}<e_{2}<\cdots<e_{N}$, then each term (except the diagonal which is one) in the expansion of determinant, $D$, of the matrix $\left(c_{i j} \mid c_{i i}\right), c_{i j}=\left|d_{i}-e_{j}\right|$, has absolute value less than one.

Proof. We proceed by induction. The lemma is vacuously true in case $N=1$. Suppose $N>1$ and let us look at a typical nonzero term of $D$. Let this term, $\pi$, contain as a factor $c_{q N} / c_{q q}$ from the $N$ th column and $c_{N p} / c_{N N}$ from the $N$ th row. Suppose that $p \neq N$, then we have $q \neq N$ and except possibly for $\operatorname{sign}\left[\pi\left(c_{q p} / c_{q q}\right)\right] /\left[\left(c_{q N} / c_{q q}\right)\left(c_{N p} / c_{N N}\right)\right]$ is a term in the expansion of the $(N-1) \times(N-1)$ determinant and hence its absolute value is no larger than one. To show $|\pi|<1$ we need only show that

$$
\begin{gathered}
\frac{c_{q N} \cdot c_{N p}}{c_{q p} \cdot c_{N N}}<1 \\
\frac{c_{q N} \cdot c_{N p}}{c_{q p} \cdot c_{N N}}=\frac{\left(e_{N}-d_{q}\right)\left(e_{p}-d_{N}\right)}{\left(e_{p}-d_{q}\right)\left(e_{N}-d_{N}\right)}<1
\end{gathered}
$$

if and only if

$$
\begin{gathered}
-d_{q} e_{p}-d_{N} e_{N}<-d_{q} e_{N}-d_{N} e_{p} \\
\left(d_{N}-d_{q}\right) e_{p}<\left(d_{N}-d_{q}\right) e_{N}
\end{gathered}
$$

$e_{p}<e_{N}$ which is one of our assumptions. If $p=N$, then $\pi$ is a term of the $(N-1) \times(N-1)$ determinant. This completes the induction.

When we have established the following lemma we can immediately conclude that $\lim _{n \rightarrow \infty} \operatorname{Det}(Q(n)-\lambda I)=(-\lambda)^{N}$.

Lemma 5.2. For a regular system $\left(\mathrm{S}_{1}\right)$ each term (except the diagonal term which is one) in the expansion of the determinant of $\left(b_{i j} / b_{i i}\right)$ where 
$b_{i j}=\left|\varphi_{\imath}^{1}(0)-A^{j}(0,0)\right|$ has absolute value less than one.

Proof. We delete the first column and row of $\left(b_{i j} / b_{i i}\right)$ and use Lemma 5.1. Suppose $\pi$ is any term of Det $\left(b_{i j} / b_{i i}\right)$. Let $\pi$ contain $b_{1 p} / b_{11}$ from the 1st row and $b_{q 1} / b_{q q}$ from the 1 st column. Suppose $p \neq 1$, then $q \neq 1$. Use Lemma 5.1 to see that

$$
\begin{gathered}
\frac{|\pi| \frac{b_{q p}}{b_{q q}}}{\frac{b_{1 p}}{b_{11}} \cdot \frac{b_{q 1}}{b_{q q}}} \leqq 1 \\
\frac{b_{1 p} \cdot b_{q 1}}{b_{q p} \cdot b_{11}}=\frac{A^{p}(0,0)-\varphi_{1}^{1}(0)}{A^{p}(0,0)-\varphi_{q}^{1}(0)} \cdot \frac{\varphi_{q}^{1}(0)-A^{1}(0,0)}{\varphi_{1}^{1}(0)-A^{1}(0,0)}<1
\end{gathered}
$$

if and only if

$$
\begin{aligned}
A^{\mathrm{p}}(0,0) \varphi_{q}^{\mathrm{l}}(0)+A^{1}(0,0) \varphi_{1}^{1}(0) & <A^{p}(0,0) \varphi_{1}^{1}(0)+A^{1}(0,0) \varphi_{q}^{1}(0) \\
{\left[A^{p}(0,0)-A^{1}(0,0)\right] \varphi_{q}^{1}(0) } & <\left[A^{p}(0,0)-A^{1}(0,0)\right] \varphi_{1}^{1}(0) \\
\varphi_{q}^{1}(0) & <\varphi_{1}^{1}(0) .
\end{aligned}
$$

In case $p=1$ Lemma 5.1 yields our result immediately.

THEOREM 5.1. If $\left(\mathrm{S}_{1}\right)$ is regular, then for any $\varepsilon>0$ there is an $\bar{n}$ such that

$$
\bar{\lambda}(Q(n))<\varepsilon \text { for all } n>\bar{n} .
$$

Let us consider systems with constant coefficients of the form

$\left(\overline{\mathbf{S}}_{0}\right)$

$$
\left\{\begin{array}{l}
U_{x}^{i}+A^{i} U_{y}^{i}=F^{i}(x, y) \\
\sum_{j=1}^{N} a_{i j} U^{i}\left(x, m_{i} x\right)=H_{i}(x), i=1, \cdots, N .
\end{array}\right.
$$

We suppose that the constants $m_{1}, \cdots, m_{N}, A^{1}, \cdots, A^{N}$ are ordered so that

$$
A^{1} \leqq m_{2} \leqq m_{3} \leqq \cdots \leqq m_{M} \leqq m_{1} \leqq A^{2} \leqq \cdots \leqq A^{n} .
$$

We have shown that $\left(\overline{\mathrm{S}}_{0}\right)$ has at most one $C^{n+1}$ solution on $R_{1}$ if $M(0)$, $\cdots, M(n-1)$ are nonsingular and $F^{i}, H^{i}$ are $C^{n+1}$ and $\bar{\lambda}(Q(n))<1$. We will now investigate to what extent these conditions for uniqueness are necessary.

In $\left(\overline{\mathbf{S}}_{0}\right)$ suppose $M(p)$ is singular for some integer $p \geqq 0$. Let $e$ be a nonzero vector such that $M(p) e=0$. Then

$$
U^{i}(x, y)=\left(y-A^{i} x\right)^{p} e_{i}, \quad i=1, \cdots, N
$$

is a nontrivial polynomial solution of 
$\left(\overline{\mathbf{S}}_{00}\right)$

$$
\left\{\begin{array}{l}
U_{x}^{i}+A^{i} U_{y}^{i}=0 \\
\sum_{j=1}^{N} a_{i j} U^{j}\left(x, m_{i} x\right)=0 .
\end{array}\right.
$$

We express this in

THEOREM 5.2. If $M(p)$ is singular for some integer $p \geqq 0$, then $\left(\overline{\mathrm{S}}_{00}\right)$ has a nontrivial polynomial solution.

It is harder to show that the condition $\bar{\lambda}(Q(n))<1$ is needed. Without loss of generality we can suppose that $a_{11}=a_{22}=\cdots=a_{N N}=1$, then Lemma 5.2 shows us that

$$
\lim _{n \rightarrow \infty} \frac{\operatorname{Det} M(n)}{\left[\varphi_{1}^{1}(0)-A^{1}(0,0)\right]^{n} \cdots\left[\varphi_{N}^{1}(0)-A^{N}(0,0)\right]^{n}}=1 .
$$

We define for all real numbers $r \geqq 0$ :

$$
\begin{aligned}
& \tilde{M}(r)_{i 1}=a_{i 1}\left(m_{i}-A^{1}\right)^{r}, i=1, \cdots, N \\
& \tilde{M}(r)_{i j}=a_{i j}\left(A^{j}-m_{i}\right)^{r}, i=1, \cdots, N, j=2, \cdots, N .
\end{aligned}
$$

Then

$$
\lim _{r \rightarrow \infty} \frac{\operatorname{Det} \tilde{M}(r)}{\left|m_{1}-A^{1}\right|^{r} \cdots\left|m_{N}-A^{N}\right|^{r}}=+1
$$

Let

$$
\widetilde{Q}(n)_{i i}=1, \widetilde{Q}(n)_{i j}=-Q(n)_{i j}, i \neq j .
$$

Since $\widetilde{Q}(n)_{i i}>0, \widetilde{Q}(n)_{i j} \leqq 0, i \neq j$, if each principal minor of $\widetilde{Q}(n)$ is positive, then each component of $\widetilde{Q}(n)^{-1}$ is nonnegative. Using this fact we prove

LEMMA 5.3. If each principal minor of $\widetilde{Q}(n)$ is positive, then $\bar{\lambda}(Q(n))<1$.

Proof. We suppose $\bar{\lambda}(Q(n)) \geqq 1$ and deduce that $\widetilde{Q}(n)^{-1}$ has at least one negative component. Let $e$ be a nonzero vector and $|\lambda| \geqq 1$ and $Q(n) e=\lambda e$. Then

$$
\begin{gathered}
|\lambda|\left|e_{i}\right| \leqq \sum_{j=1}^{N} Q(n)_{i j}\left|e_{j}\right| \\
\left|e_{i}\right|-\sum_{j=1}^{N} Q(n)_{i j}\left|e_{j}\right| \leqq-(|\lambda|-1)\left|e_{i}\right| \\
\sum_{j=1}^{N} \widetilde{Q}(n)_{i j}\left|e_{j}\right| \leqq-(|\lambda|-1)\left|e_{i}\right| \leqq 0
\end{gathered}
$$




$$
f_{i}=\sum_{j=1}^{N} \widetilde{Q}(n)_{i j}\left|e_{j}\right|
$$

Since $-f_{i} \geqq 0$, if $\widetilde{Q}(n)^{-1}$ had no negative components each component of $\widetilde{Q}(n)^{-1}(-f)$ would be nonnegative. But then $\widetilde{Q}(n)^{-1} f=|e| \leqq 0$ implies that $e=0$. This is countrary to our assumption that $e \neq 0$.

We call a system $\left(\mathrm{S}_{0}\right)$ uniform if each term (except the diagonal which is one) in the expansion of $\operatorname{Det}(M(0))$ is either negative or zero. We have assumed that $a_{i i}=1$. Any $\left(\mathrm{S}_{0}\right)$ with $N=3$ satisfying $a_{13} \leqq$ $0, a_{31} \leqq 0, a_{12} \geqq 0, a_{21} \geqq 0, a_{23} \geqq 0, a_{32} \geqq 0$ is uniform.

Lemma 5.4. If $\left(\mathrm{S}_{0}\right)$ is uniform and $\operatorname{Det}(\widetilde{M}(n))>0$, then each principal minor of $\widetilde{Q}(n)$ is positive.

Combining the last two lemmas we have

LemmA 5.5. If $\left(\mathrm{S}_{0}\right)$ is uniform and $\bar{\lambda}(Q(n)) \geqq 1$, then $\operatorname{Det}(\tilde{M}(n)) \leqq 0$. Now Det $(\widetilde{M}(n))$ is eventually positive and $\operatorname{Det}(\widetilde{M}(r))$ is a continuous function of $r$.

If $\operatorname{Det}(\widetilde{M}(r))=0$, then $\widetilde{M}(r) e=0$ for some $e \neq 0$ and

$$
\begin{aligned}
& U^{1}(x, y)=\left(y-A^{1} x\right)^{r} e_{1} \\
& U^{i}(x, y)=\left(A^{i} x-y\right)^{r} e_{i}, i=2, \cdots, N
\end{aligned}
$$

is a $C^{[r]}$ solution of $\left(\mathrm{S}_{00}\right)$. We have proved

THEoREM 5.3. If $\left(\overline{\mathrm{S}}_{0}\right)$ is uniform and $\bar{\lambda}(Q(n)) \geqq 1$, then $\left(\overline{\mathrm{S}}_{00}\right)$ has a nontrivial solution which is $C^{n}$.

We can give a more complete analysis of $\left(\overline{\mathrm{S}}_{0}\right)$ when $N=2$ :

$\left(\mathrm{S}_{00}\right)$

$$
\left\{\begin{array}{l}
U_{x}^{i}+A^{i} U_{y}^{2}=0, i=1,2 \\
U^{1}\left(x, m_{1} x\right)+a U^{2}\left(x, m_{1} x\right)=0 \\
U^{1}\left(x, m_{2} x\right)+U^{2}\left(x, m_{2} x\right)=0
\end{array}\right.
$$

where $A^{1}<m_{2}<m_{1}<A^{2}$.

The eigenvalues of $M(n)$ satisfy the equation

$$
\lambda^{2}=|a| \rho^{n} \quad \text { where } \quad \rho=\frac{A^{2}-m_{1}}{m_{1}-A^{1}} \frac{m_{2}-A^{1}}{A^{2}-m_{2}} .
$$

Since $\left(\mathrm{S}_{00}\right)$ is regular, $\rho<1$.

If we had allowed $m_{2}=A^{1}$ or $m_{1}=A^{2}$ we would have had $\rho=0$. Let $r$ be the real number such that $|a| \rho^{r}=1$. Suppose that $r \geqq 1$. As we know, if $|a|<1$, then $\left(\mathrm{S}_{00}\right)$ has only the trivial solution. Let $[r]$ be the greatest integer less than or equal to $r$. Two cases arise. First if $a>0$, then $a \cdot \rho^{r}=+1$. In this case 


$$
\begin{aligned}
& U^{1}(x, y)=-\left(A^{2}-m_{2}\right)^{r}\left(y-A^{1} x\right)^{r} \\
& U^{2}(x, y)=\left(m_{2}-A^{1}\right)^{r}\left(A^{2} x-y\right)^{r}
\end{aligned}
$$

is a nontrivial $C^{[r]}$ solution of $\left(\mathrm{S}_{00}\right)$. If $r$ is an integer these functions are polynomials. In this connection we notice that $M(n)$ is singular just in case $1-a \rho^{n}=0$ which can happen only if $r$ is an integer and $a>0$. Now suppose $a$ is negative, then $a \rho^{r}=-1$ and

$$
\begin{aligned}
& U^{1}(x, y)=\left(A^{2}-m_{2}\right)^{r}\left(y-A^{1} x\right)^{r} \sin \left(\frac{\log \frac{y-A^{1} x}{m_{1}-A^{1}}}{\log \rho}\right) \pi \\
& U^{2}(x, y)=\left(m_{2}-A^{1}\right)^{r}\left(A^{2} x-y\right)^{r} \sin \left(\frac{\log \frac{A^{2} x-y}{A^{2}-m_{1}}}{\log \rho}\right) \pi
\end{aligned}
$$

is a nontrivial $C^{[r]}$ solution of $\left(\mathrm{S}_{00}\right)$.

6. Application to second order equations. We apply our results to the 2nd order system $\left(\mathrm{S}_{2}\right)$. Our method is however equally suited for the $n$th order case.

The system

$\left(\mathbf{S}_{2}\right)\left\{\begin{array}{l}Z_{x x}-Z_{y y}=A(x, y) Z_{x}+B(x, y) Z_{y}+C(x, y) Z+D(x, y) \\ b_{i 1}(x) Z_{x}\left(x, \varphi_{\imath}(x)\right)+b_{i 2}(x) Z_{y}\left(x, \varphi_{i}(x)\right)+b_{i 3}(x) Z\left(x, \varphi_{i}(x)\right)=H_{i}(x), i=1,2 \\ Z(0,0)=c\end{array}\right.$ is transformed into the equivalent system

$$
\left\{\begin{array}{l}
U_{x}-U_{y}=1 / 2(A+B) U+1 / 2(-A+B) V+C Z+D \\
V_{x}+V_{y}=-1 / 2(A+B) U-1 / 2(-A+B) V-C Z-D \\
\left(b_{i 1}+b_{i 2}\right) U\left(x_{1} \varphi_{i}(x)\right)+\left(-b_{i 1}+b_{i 2}\right) V\left(x, \varphi_{i}(x)\right) \\
\quad=-2 b_{i 3} Z\left(x, \varphi_{i}(x)\right)+2 H_{i}(x) \quad i=1,2 \\
Z_{y}=1 / 2(U+V), Z_{x}=1 / 2(U-V) \\
Z(0,0)=c
\end{array}\right.
$$

by the substitution $U=Z_{x}+Z_{y}$ and $V=-Z_{x}+Z_{y}$.

If we iterate as follows:

$$
\begin{gathered}
U_{x}^{s+1}-U_{y}^{s+1}=1 / 2(A+B) U^{s}+1 / 2(-A+B) V^{s}+C Z^{s}+D \\
V_{x}^{s+1}+V_{y}^{s+1}=-1 / 2(A+B) U^{s}-1 / 2(-A+B) V^{s}-C Z^{s}-D \\
\left(b_{i 1}+b_{i 2}\right) U^{s+1}\left(x, \varphi_{i}(x)\right)+\left(-b_{i 1}+b_{i 2}\right) V^{s+1}\left(x, \varphi_{i}(x)\right) \\
=-2 b_{i 3} Z^{s}\left(x, \varphi_{i}(x)\right)+2 H_{i}(x) \\
Z_{y}^{s+1}=1 / 2\left(U^{s+1}+V^{s+1}\right), Z_{x}^{s+1}=1 / 2\left(U^{s+1}-V^{s+1}\right)
\end{gathered}
$$




$$
Z^{s+1}(0,0)=c
$$

and let $a_{11}=b_{11}+b_{12}, a_{12}=-b_{11}+b_{12}, a_{21}=b_{21}+b_{22}, a_{22}=-b_{21}+b_{32}, A^{1}=$ $-1, A^{2}=+1$ we have using the same methods as in $\S 3$.

Lemma 6.1. If $n$ is any nonnegative integer and $A, B, C, D, b_{i j}$, $H_{i}, \varphi_{i}$ are $C^{n+1}$ and $\bar{\lambda}(Q(n))<1$ and $\bar{\lambda}(Q(n+1))<1$ and $M(0), \cdots$, $M(n-1)$ are nonsingular, then on some $R_{1}$ there is exactly one $C^{n+1}$ solution of $\left(\mathrm{S}_{2}\right)$.

If we assume that $-1 \leqq \varphi_{:}^{\prime}(0)<\varphi_{1}^{\prime}(0) \leqq 1$ and let

$$
\begin{aligned}
& a=\frac{-b_{11}(0)+b_{12}(0)}{b_{11}(0)+b_{12}(0)}, \quad b=\frac{b_{21}(0)+b_{22}(0)}{-b_{21}(0)+b_{22}(0)}, \\
& r=\frac{1-\varphi_{1}^{\prime}(0)}{1+\varphi_{1}^{\prime}(0)} \cdot \frac{1+\varphi_{2}^{\prime}(0)}{1-\varphi_{2}^{\prime}(0)}\{\text { Notice that } 0 \leqq r<1]
\end{aligned}
$$

we have immediately

THeOREM 6.1. If $n$ is a nonnegative integer and $|a b| r^{n}<1$ and $a b r^{k} \neq 1$ for $k=0, \cdots, n-1$ and $A, B, C, D, b_{i j}, H_{i}, \varphi_{\imath}$ are $C^{n+1}$, then on some $R_{1}$ there is exactly one $C^{n+1}$ solution of $\left(\mathrm{S}_{2}\right)$.

Since $0 \leqq r<1$ there always is a nonnegative integer such that

$$
|a b| \cdot r^{n}<1 \text {. }
$$

It is interesting to notice that if

$a b \cdot r^{p}=1$ for some $p \geqq 1$ which need not be an integer, then

$$
Z(x, y)=\left(1-m_{2}\right)^{p} \frac{(x+y)^{p+1}}{p+1}+b\left(1+m_{2}\right)^{p} \frac{(x-y)^{p+1}}{p+1}
$$

is a non-trivial solution of

$$
\begin{aligned}
& Z_{x x}-Z_{y y}=0 \\
& b_{i 1} Z_{x}\left(x, m_{i} x\right)+b_{i 2} Z_{y}\left(x, m_{i} x\right)=0, i=1,2 \\
& Z(0,0)=0 .
\end{aligned}
$$

This $Z$ is a polynomial in case $p$ is an integer.

We finish by applying our theorem to a problem solved by Goursat [2]:

$$
\begin{aligned}
& Z_{x x}-Z_{y y}=A Z_{x}+B Z_{y}+D \\
& Z\left(x, m_{i} x\right)=H_{i}(x), i=1,2 \text { where } \\
& H_{1}(0)=H_{2}(0),-1 \leqq m_{2}<m_{1} \leqq 1 .
\end{aligned}
$$

An equivalent problem is 


$$
\begin{aligned}
& Z_{x x}-Z_{y y}=A Z_{x}+B Z_{y}+C Z+D \\
& Z_{x}\left(x, m_{i} x\right)+m_{i} Z_{y}\left(x, m_{i} x\right)=H_{i}^{\prime}(x), \quad i=1,2 \\
& Z(0,0)=H_{i}(0) .
\end{aligned}
$$

We have in this case

$$
|a b|=\frac{1-m_{1}}{1+m_{1}} \cdot \frac{1+m_{2}}{1-m_{2}}=r<1 .
$$

Consequently according to Theorem 6.1 this problem has exactly one $C^{1}$ solution.

In [1] the authors treat a somewhat more general system the functions of which satisfy certain Lipschitz conditions. They make in our notation the hypotheses $|a b|<1$ and $r<1$. In [8] Szmydt solves the same problem with the hypothesis that some of the Lipschitz constants are small. The result is essentially the same.

\section{BIBLIOGRAPHY}

1. A. Bielecki, et J. Kisynski, Sur un probleme de Mlle Z. Szmydt relatif a l'equation $\partial^{2} z / \partial x \partial y=f[x, y, z,(\partial z, / \partial x, \partial z / \partial y)]$, Bull. Acad. Polon. Sci. Ser. Sci. Math. Astr. Phys., 6 (1958), 321-325.

2. E. Goursat, Sur un procede alterne, C. R. Acad. Sci. Paris, 149 (1909), 762-765.

3. R. P. Holten, Generalized Goursat problem for a hyperbolic system, Technical Report no. 2, AFOSR RN 58-1008, AF 49(638)-398, (1958).

4. L. A. Meltzer, On the correct statement of Goursat's problem, Recueil Mathematique, 60 (1946), 59-101.

5. V. P. Mihailov, Non-analytic solutions of Goursat's problem for a system of differential equations in two independent variables, Dokl. Akad. Nauk SSSR (N.S.) 117 (1957), 759-762. 6. G. Peyser, Energy integrals for the mixed problem in hyperbolic partial differential equations of higher order, Journ. Math. and Mech., 6 (1957), 641-653.

7. E. Picard, Memoire sur la theorie des equations aux derivees partielles et la methode des approximations successives, Journ. de Math. 4e serie t. VI (1890), 145-210.

8. Z. Szmydt, Sur l'existence de solutions de certains problems aux limites relatifs a un systeme d'equations differentielles hyperboliques, Bull. Acad. Polon. Sci. Ser. Sci. Math. Astr. Phys., 6 (1958), 31-36.

9. Setuzo Yosida, Hukuhara's problem for hyperbolic equations with two independent variables I. Semi-linear case, Proc. Japan Acad. 34 (1958), 319-324. 


\section{PACIFIC JOURNAL OF MATHEMATICS}

\section{EDITORS}

Ralph S. Phillips

Stanford University

Stanford, California

M. G. Arsove

University of Washington

Seattle 5, Washington
A. L. Whiteman

University of Southern Californla

Los Angeles 7, California

Lowell J. Paige

University of California

Los Angeles 24, California

\section{ASSOCIATE EDITORS}

E. F. BECKENBACH

D. DERRY

H. L. ROYDEN

E. G. STRAUS

T. M. CHERRY

M. OHTSUKA

E. SPANIER

F. WOLF

\section{SUPPORTING INSTITUTIONS}

UNIVERSITY OF BRITISH COLUMBIA

STANFORD UNIVERSITY

CALIFORNIA INSTITUTE OF TECHNOLOGY

UNIVERSITY OF CALIFORNIA

MONTANA STATE UNIVERSITY

UNIVERSITY OF TOKYO

UNIVERSITY OF UTAH

UNIVERSITY OF NEVADA

NEW MEXICO STATE UNIVERSITY

OREGON STATE UNIVERSITY

UNIVERSITY OF OREGON

OSAKA UNIVERSITY

WASHINGTON STATE UNIVERSITY

UNIVERSITY OF WASHINGTON

UNIVERSITY OF SOUTHERN CALIFORNIA

AMERICAN MATHEMATICAL SOCIETY CALIFORNIA RESEARCH CORPORATION SPACE TECHNOLOGY LABORATORIES NAVAL ORDNANCE TEST STATION 


\section{Pacific Journal of Mathematics}

\section{Vol. 12, No. $1 \quad$ January, 1962}

Jonathan L. Alperin, Groups with finitely many automorphisms $\ldots \ldots \ldots \ldots \ldots \ldots \ldots \ldots$

Martin Arthur Arkowitz, The generalized Whitehead product ................ 7

John D. Baum, Instability and asymptoticity in toplogical dynamics . . . . . . . . . . 25

William Aaron Beyer, Hausdorff dimension of level sets of some Rademacher series .... $\quad 35$

Frank Herbert Brownell, III, A note on Cook's wave-matrix theorem . . . . . . . . . . . . . 47

Gulbank D. Chakerian, An inequality for closed space curves ................. 53

Inge Futtrup Christensen, Some further extensions of a theorem of Marcinkiewicz ....... 59

Charles Vernon Coffman, Linear differential equations on cones in Banach spaces . . . . . 69

Eckford Cohen, Arithmetical notes. III. Certain equally distributed sets of integers . . . . . 77

John Irving Derr and Angus E. Taylor, Operators of meromorphic type with multiple poles

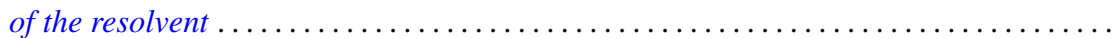

Jacob Feldman, On measurability of stochastic processes in products space .............

Robert S. Freeman, Closed extensions of the Laplace operator determined by a general class of boundary conditions, for unbounded regions ......................

Robert E. Fullerton, Geometric structure of absolute basis systems in a linear topological

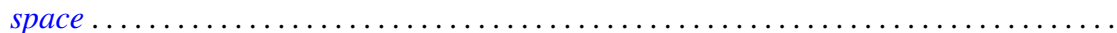

Dieter Gaier, On conformal mapping of nearly circular regions

Andrew Mattei Gleason and Hassler Whitney, The extension of linear functionals defined

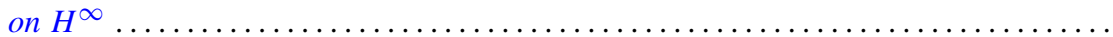

Seymour Goldberg, Closed linear operators and associated continuous linear

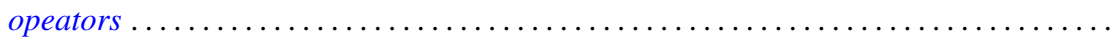

Basil Gordon, Aviezri Siegmund Fraenkel and Ernst Gabor Straus, On the determination of sets by the sets of sums of a certain order

Branko Grünbaum, The dimension of intersections of convex sets. .

Paul Daniel Hill, On the number of pure subgroups

Robert Peter Holten, Generalized Goursat problem . .

Alfred Horn, Eigenvalues of sums of Hermitian matrices ...........

Henry C. Howard, Oscillation and nonoscillation criteria for

$$
y^{\prime \prime}(x)+f(y(x)) p(x)=0
$$

Taqdir Husain, $S$-spaces and the open mapping theorem ...

Richard Eugene Isaac, Markov processes and unique stationary probability measures ...

John Rolfe Isbell, Supercomplete spaces ....................

John Rolfe Isbell, On finite-dimensional uniform spaces. II .........

N. Jacobson, A note on automorphisms of Lie algebras ..............

Antoni A. Kosinski, A theorem on families of acyclic sets and its applications

Marvin David Marcus and H. Minc, The invariance of symmetric functions of singular values...

Ralph David McWilliams, A note on weak sequential convergence.

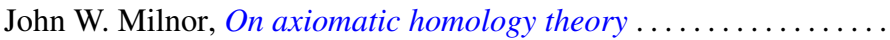

Victor Julius Mizel and Malempati Madhusudana Rao, Nonsymmetric projections in

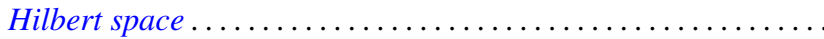

Calvin Cooper Moore, On the Frobenius reciprocity theorem for locally compact

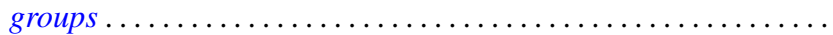

Donald J. Newman, The Gibbs phenomenon for Hausdorff means . 